12. Simon, D.B., et al. 1999. Paracellin-1, a renal tight junction protein required for paracellular $\mathrm{Mg} 2+$ resorption [see comments]. Science. 285:103-106.

13. Knoers, N.V. 2009. Inherited forms of renal hypomagnesemia: an update. Pediatr. Nephrol. 24:697-705.

14. Xu, J.Z., et al. 1997. Localization of the ROMK protein on the apical membranes of rat kidney neph- ron segments. Am. J. Physiol. 273:F739-F748

15. Vacher, H., Mohapatra, D.P., and Trimmer, J.S 2008. Localization and targeting of voltage-dependent ion channels in mammalian central neurons. Physiol. Rev. 88:1407-1447.

16. Milionis, H.J., et al. 2002. Hypomagnesemia and concurrent acid-base and electrolyte abnormalities in patients with congestive heart failure. Eur. J.
Heart Fail. 4:167-173

17. Allen, G.G., and Barratt, L.J. 1985. Effect of cisplatin on the transepithelial potential difference of rat distal tubule. Kidney Int. 27:842-847.

18. Ellison, D.H. 2000. Divalent cation transport by the distal nephron: insights from Bartter's and Gitelman's syndromes. Am. J. Physiol. Renal Physiol. 279:F616-F625.

\title{
Myoglobin tames tumor growth and spread
}

\author{
Ulrich Flögel ${ }^{1}$ and Chi V. Dang ${ }^{2}$
}

IInstitut für Herz- und Kreislaufphysiologie, Heinrich-Heine-Universität, Düsseldorf, Germany. 2Division of Hematology, Department of Medicine, Johns Hopkins University School of Medicine, Baltimore, USA.

Tumor growth is accompanied by tissue hypoxia, but does this reduced oxygen availability promote further tumor expansion, resulting in a vicious cycle? In this issue of the JCI, Galluzzo et al. report that increasing oxygen tension in tumor cells by ectopically expressing the oxygen-binding hemoprotein myoglobin indeed affects tumorigenesis (see the related article beginning on page 865). Tumors derived from cells transfected with myoglobin grew more slowly, were less hypoxic, and were less metastatic. These results will spur further mechanistic inquiry into the role of hypoxia in tumor expansion.

Tumorigenesis involves not only cell autonomous genetic alterations that result in activation of oncogenes and loss of tumor suppressor genes but also adaptation of neoplastic cells to the tumor microenvironment. Although the activation of oncogenes and their products MYC, AKT, $\mathrm{PI} 3 \mathrm{~K}$, and RAS as well as the loss of p53 and VHL tumor suppressors have been linked to altered tumor metabolism, it has become apparent that the hypoxic tumor microenvironment, which activates the low oxygen-sensing HIF transcription factors, also plays a key role in tumor metabolism and tumorigenesis (1). It is well documented that new blood vessels recruited into a growing tumor mass are disorganized (2), often culminating in vascular "deadends" rather than providing the canonical microvasculature characteristic of normal tissue, in which arterioles are connected to venules via a capillary bed. Hence, tumors endure significant hypoxia that is distributed heterogeneously within a tumor mass, and areas of tumor hypoxia could fluctuate with time (3). In this regard, whether tumor

Conflict of interest: The authors have declared that no conflict of interest exists.

Nonstandard abbreviations used: $\mathrm{Cb}$, cytoglobin; $\mathrm{Mb}$, myoglobin.

Citation for this article: J. Clin. Invest. 119:766-768 (2009). doi:10.1172/JCI38796. hypoxia itself contributes to tumor progression has been a matter of debate rather than of experimentation.

\section{Transduction of myoglobin to improve tumor oxygenation}

In this issue of the JCI, Galluzzo et al. (4) present an innovative and elegant approach to addressing the question of whether hypoxia is a side effect of or a key player in tumor progression. The authors used lentiviral vector-mediated transduction to achieve expression of myoglobin $(\mathrm{Mb})$ in human lung carcinoma cells as a genetic tool to prevent tumor hypoxia. Like its molecular relative hemoglobin, $\mathrm{Mb}-\mathrm{a}$ cytosolic hemoprotein present in skeletal and heart muscle (5) - reversibly binds $\mathrm{O}_{2}$ and thus facilitates $\mathrm{O}_{2}$ transport from the blood to mitochondria during periods of increased metabolic activity or serves as an $\mathrm{O}_{2}$ reservoir under hypoxic conditions (Figure 1). Transduced cancer cells expressing $\mathrm{Mb}$ showed no signs of altered cellular proliferation in vitro, but, surprisingly, in vivo xenograft growth was severely diminished after injection of transduced cells into mice. The data reported by Galluzzo and coworkers are quite impressive: presence of $\mathrm{Mb}$ resulted in a 5 -fold decrease in tumor expansion compared with controls. Furthermore, the expression of $\mathrm{Mb}$ suppressed both local and distal metastatic spread, led to enhanced cancer cell differentiation, and reduced the degree of abnormal vascularization. The authors also report that the expression of HIF-1 $\alpha$, which is generally accepted as a master regulator of the cellular hypoxic response, was downregulated in $\mathrm{Mb}$-expressing cancer cells. The data suggest that the beneficial effects of the presence of $\mathrm{Mb}$ within cancer cells are the result of improved $\mathrm{O}_{2}$ delivery to the tumor, which "calms" the tumor's craving to expand.

\section{Globins, oxygen, and beyond}

Beyond the initial finding of $\mathrm{Mb}^{\prime} \mathrm{O}_{2}$-binding properties - set into a physiological context especially by the seminal work of Beatrice and Jonathan Wittenberg - within the last decade, the field of globin biology was invigorated, particularly by the generation of gene-deficient mutants but also by the discovery of two new members of the globin family, cytoglobin $(\mathrm{Cb})$ and neuroglobin (reviewed in refs. 5-8). Based on this recent work, the role of $\mathrm{Mb}$ in muscle physiology has been reassessed and its scope of function has been considerably extended beyond oxygen storage and delivery to also include a role as an important scavenger of NO and ROS, signaling molecules involved in cellular oxidative stress (Figure 1). Appreciating the diversity of Mb's properties, Galluzzo et al. (4) used mutated forms of $\mathrm{Mb}$ unable to bind $\mathrm{O}_{2}$ to demonstrate that the inhibition of tumor progression is primarily caused by improved $\mathrm{O}_{2}$ delivery. However, it should be kept in mind that structural alterations at Mb's hemebinding pocket will also alter the in vivo kinetics for all other events taking place at this site, including radical reactions. It is, therefore, tempting to speculate that these newly uncovered properties of $\mathrm{Mb}$ may 


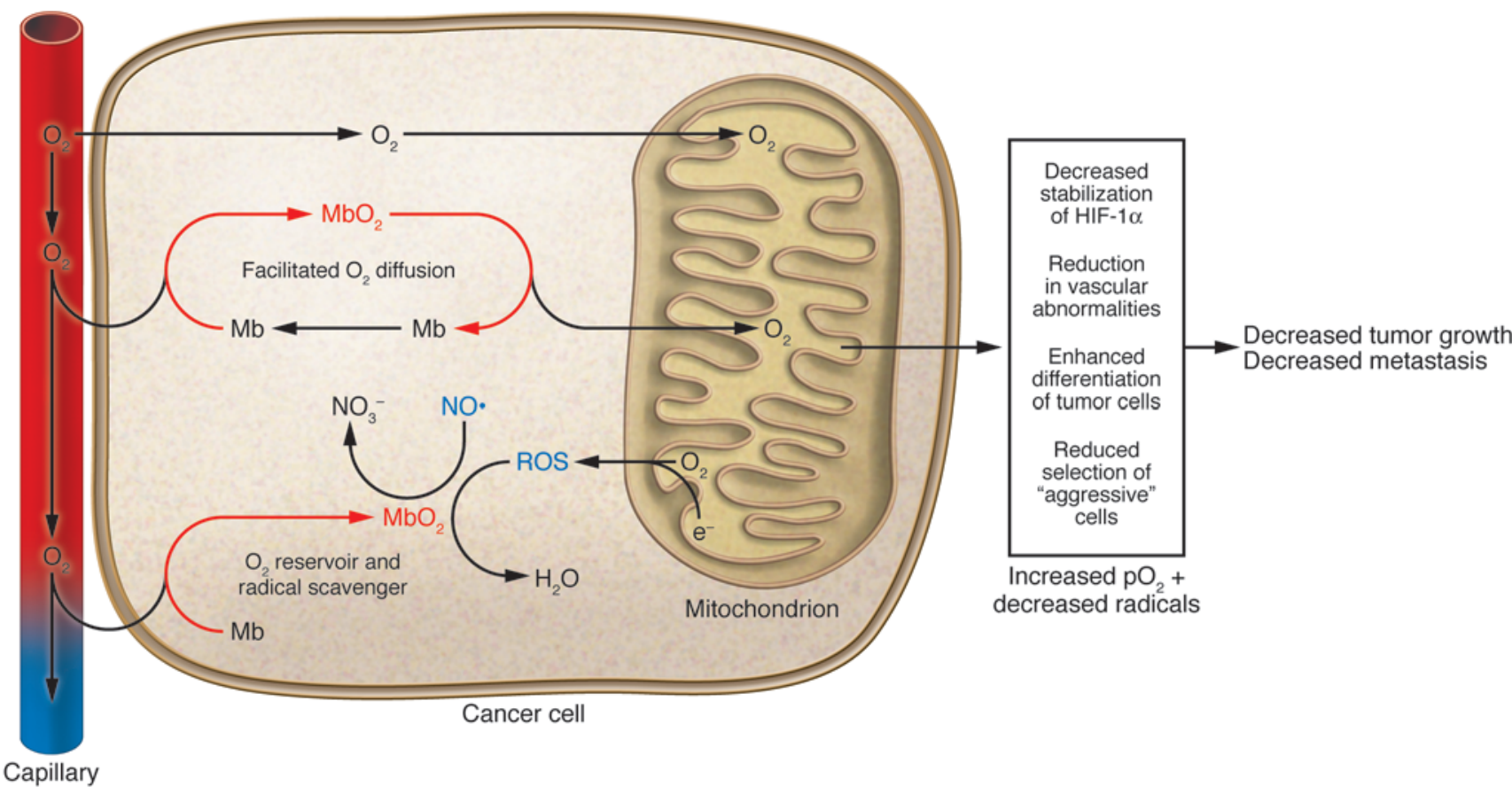

Figure 1

Possible interactions of $\mathrm{Mb}$ within the cancer cell and the metabolic and functional consequences on tumor growth and spread. Tumor progression is well known to be associated with hypoxia, which itself gives rise to enhanced stabilization of the master regulator HIF-1 $\alpha$ and also to an augmented release of ROS, caused by an increased leakage of the mitochondrial electron transport chain. Due to its high $\mathrm{O}_{2}$ affinity, $\mathrm{Mb}$ may serve as an $\mathrm{O}_{2}$ store and facilitate $\mathrm{O}_{2}$ transport from capillaries to mitochondria even under conditions of limited $\mathrm{O}_{2}$ availability. As a consequence, the ectopic expression of $\mathrm{Mb}$ within cancer cells carried out by Galluzzo et al. (4) leads to an improved $\mathrm{O}_{2}$ supply to the tumor, as reflected by the enhanced tissue $\mathrm{O}_{2}$ partial pressure $\left(\mathrm{pO}_{2}\right)$ reported in this study. Additionally, $\mathrm{Mb}$ can reduce oxidative and nitrosative stress in cancer cells by detoxification of ROS to water and of $\mathrm{NO}$ radicals (NO-) to nitrate $\left(\mathrm{NO}_{3}{ }^{-}\right)$, respectively. Together, these result in an environment of enhanced aerobic mitochondrial respiration and reduced levels of free radicals, favoring efficient degradation of HIF-1 $\alpha$, a key player in the progression of tumor metabolism to a more aggressive phenotype (15). As demonstrated by Galluzzo et al. (4), the metabolic environment created by the presence of $\mathrm{Mb}$ in cancer cells furthermore implicates cell differentiation, a reduction in vascular abnormalities, and a reduced selection of aggressive cells, which, in turn, retards tumor growth and metastatic spread.

contribute or even account in a synergistic manner for the inhibition of tumor growth and spreading. Although the role of $\mathrm{NO}$ in cancer is far from clear (9), there is evidence that, especially during the early phase of tumor development, NO may contribute to tumor promotion and progression by stabilizing HIF-1 $\alpha$ (10), thereby mimicking hypoxia and thus initiating a genetic program that helps the tumor to survive and grow, in part by stimulating angiogenesis. Since HIF-1 $\alpha$ is similarly stabilized by enhanced ROS levels (11), scavenging of these species not only reduces the nitrosative and oxidative stress but also prevents activation of a signal cascade that would prepare the ground for tumor expansion.

In addition to the functions mentioned by Galluzzo and coworkers (4), a lesser known property of $\mathrm{Mb}$ is its ability to bind fatty acids. Therefore, Mb has been suggested as serving as an intracellular fattyacid transporter (12) working in support of the well-known fatty acid-binding protein, which is generally assumed to be the major player in fatty-acid delivery to mitochondria. Interestingly, fatty-acid binding of $\mathrm{Mb}$ depends on its oxygenation state, in that conformational changes caused by binding of $\mathrm{O}_{2}$ promote the interaction of $\mathrm{Mb}$ with fatty acids (13). Although the functional relevance of fatty-acid binding to $\mathrm{Mb}$ remains to be explored, the simultaneous delivery of $\mathrm{O}_{2}$ and fatty acids to mitochondria would clearly favor true aerobic respiration. According to this hypothesis, the presence of $\mathrm{Mb}$ would diminish the need of tumor cells to rely on inefficient aerobic glycolysis and thereby prevent a metabolic state that has been proposed to facilitate the expansion of cancer cells (14).

When these data are considered together, it seems likely that, in concert, the multifaceted properties of $\mathrm{Mb}$ create an environment characterized by improved aerobic mitochondrial respiration and low levels of free radicals. This results in enhanced degradation of HIF- $1 \alpha$, considered to be a master regulator in the progression of tumor metabolism to a more aggressive phenotype (15). As a consequence, the presence of $\mathrm{Mb}$ within cancer cells favors cell differentiation, reduces tumor expansion, and suppresses metastatic spread, as convincingly demonstrated by the comprehensive investigations reported in this issue by Galluzzo et al. (4). In this context, it is noteworthy that - as also mentioned by the authors - the newest member of the globin family, $\mathrm{Cb}$, has been recently identified as a tumor suppressor (16). However, since the specific functions of $\mathrm{Cb}$ are still a matter of intense debate (8), the underlying mechanisms of this effect have yet to be scrutinized. Nevertheless, these observations are exciting per se, and it will be absorbing to follow how these relationships can be transferred into a clinical context or utilized for future drug design or therapy. 


\section{Oxygen availability and tumors: a tricky relationship}

The findings of Galluzzo et al. (4) not only provide additional insights into the role of the tumor microenvironment and oxygen in tumorigenesis, but they also raise additional questions regarding our understanding of oxygen metabolism and tumorigenesis. For example, it has been documented that high-altitude populations, subject to chronic hypoxia, tend to have a lower frequency of cancer (17). Is this because there is an adaptation that increases oxygen transport into tissues (e.g., enhanced $\mathrm{Mb}$ expression; ref. 18) or because oxygen deprivation is antitumorigenic? In addition, it is known that animals with larger body mass tend to have a lower incidence of cancer development (19), and yet the tissues from these animals have lower specific metabolic rates and oxygen consumption (20). Notwithstanding these intriguing nuances, the work of Galluzzo and coworkers clearly provides additional food for our collective thought on tumor metabolism and tumorigenesis.

Address correspondence to: Ulrich Flögel, Institut für Herz- und Kreislaufphysiologie, Heinrich-Heine-Universität,
Universitätsstrasse 1, 40225 Düsseldorf, Germany. Phone: 49-211-8112785; Fax: 49211-8112672; E-mail: floegel@uni-duesseldorf.de. Or to: Chi Dang, Johns Hopkins Medicine, Ross Building, Room 1032, 720 Rutland Avenue, Baltimore, Maryland 21205. Phone: (410) 955-2411; Fax: (410) 955-0185; E-mail: cvdang@jhmi.edu.

1. Hsu, P.P., and Sabatini, D.M. 2008. Cancer cell metabolism: Warburg and beyond. Cell. 134:703-707.

2. Jain, R.K. 2005. Normalization of tumor vasculature: an emerging concept in antiangiogenic therapy. Science. 307:58-62.

3. Dewhirst, M.W., Cao, Y., and Moeller, B. 2008. Cycling hypoxia and free radicals regulate angiogenesis and radiotherapy response. Nat. Rev. Cancer. 8:425-437.

4. Galluzzo, M., Pennacchietti, S., Rosano, S., Comoglio, P.M., and Michieli, P. 2009. Prevention of hypoxia by myoglobin expression in human tumor cells promotes differentiation and inhibits metastasis. J. Clin. Invest. 119:865-875.

5. Wittenberg, J.B., and Wittenberg, B.A. 2003. Myoglobin function reassessed. J. Exp. Biol. 206:2011-2020.

6. Ordway, G.A., and Garry, D.J. 2004. Myoglobin: an essential hemoprotein in striated muscle. J. Exp. Biol. 207:3441-3446.

7. Gödecke, A. 2006. On the impact of NO-globin interactions in the cardiovascular system. Cardiovasc. Res. 69:309-317.

8. Burmester, T., Gerlach, F., and Hankeln, T. 2007. Regulation and role of neuroglobin and cytoglobin under hypoxia. Adv. Exp. Med. Biol. 618:169-180.

9. Wink, D.A., Ridnour, L.A., Hussain, S.P., and Harris, C.C. 2008. The reemergence of nitric oxide and cancer. Nitric Oxide. 19:65-67.

10. Quintero, M., Brennan, P.A., Thomas, G.J., and Moncada, S. 2006. Nitric oxide is a factor in the stabilization of hypoxia-inducible factor-1alpha in cancer: role of free radical formation. Cancer Res. 66:770-774.

11. Simon, M.C. 2006. Mitochondrial reactive oxygen species are required for hypoxic HIF alpha stabilization. Adv. Exp. Med. Biol. 588:165-170.

12. Gloster, J., and Harris, P. 1977. Fatty acid binding to cytoplasmic proteins of myocardium and red and white skeletal muscle in the rat. A possible new role for myoglobin. Biochem. Biophys. Res. Commun. 74:506-513.

13. Götz, F.M., Hertel, M., and Groschel-Stewart, U. 1994. Fatty acid binding of myoglobin depends on its oxygenation. Biol. Chem. Hoppe Seyler. 375:387-392.

14. Gatenby, R.A., and Gillies, R.J. 2004. Why do cancers have high aerobic glycolysis? Nat. Rev. Cancer. 4:891-899.

15. Dang, C.V., Kim, J.W., Gao, P., and Yustein, J. 2008. The interplay between MYC and HIF in cancer. Nat. Rev. Cancer. 8:51-56.

16. Shivapurkar, N., et al. 2008. Cytoglobin, the newest member of the globin family, functions as a tumor suppressor gene. Cancer Res. 68:7448-7456.

17. Weinberg, C.R., Brown, K.G., and Hoel, D.G. 1987. Altitude, radiation, and mortality from cancer and heart disease. Radiat. Res. 112:381-390.

18. Terrados, N. 1992. Altitude training and muscular metabolism. Int. J. Sports Med. 13(Suppl. 1): S206-S209.

19. Nagy, J.D., Victor, E.M., and Cropper, J.H. 2007. Why don't all whales have cancer? A novel hypothesis resolving Peto's paradox. Integr. Comp. Biol. 47:317-328. doi:10.1093/icb/icm062.

20. Savage, V.M., et al. 2007. Scaling of number, size, and metabolic rate of cells with body size in mammals. Proc. Natl. Acad. Sci. U. S. A. 104:4718-4723.

\section{Kallikreins and lupus nephritis}

\section{Claudio Ponticelli' and Pier Luigi Meroni²}

${ }^{1}$ Division of Nephrology, IRCCS Istituto Clinico Humanitas, Milan, Italy. ${ }^{2}$ Clinical Immunology and Rheumatology Unit, Department of Internal Medicine, IRCCS Istituto Auxologico Italiano, University of Milan, Milan, Italy.

The kidney kallikrein-kinin system plays important roles in inflammation, coagulation, angiogenesis, and regulation of vessel tone and permeability. In this issue of the JCI, Liu et al. provide data that suggest a protective role for kallikrein in animal models of anti-glomerular basement membrane (GBM) antibody-induced nephritis, an experimental model of Goodpasture disease (see the related article beginning on page 911). Furthermore, human systemic lupus erythematosus and lupus nephritis were shown to be associated with kallikrein 1 (KLK1) and the $K L K 3$ promoter. The authors suggest that kallikrein genes are involved in the development of SLE and lupus nephritis and may exert a renoprotective role. It is possible, however, that the kallikrein-kinin system may play dual roles: protecting the kidney against ischemia and interstitial fibrosis while also mediating vasodilation, inflammation, and activation of the innate immune response.

Conflict of interest: C. Ponticelli is a consultant for Novartis, Italy.

Nonstandard abbreviations used: ACE, angiotensin converting enzyme; GBM, glomerular basement membrane; KLK1, kallikrein 1.

Citation for this article: J. Clin. Invest. 119:768-771 (2009). doi:10.1172/JCI38786.

\section{The kallikrein-kinin system}

The kallikrein-kinin system comprises proteins that play a role in inflammation, coagulation, and regulation of vessel tone and permeability via the production of small peptides called kinins. The system originates from prekallikrein, a serum protease that, after being activated by factor XIIa (also known as Hageman factor), is cleaved to form kallikrein. Kallikreins are serine peptidases (kininogenases) that rapidly release kinins in the plasma by cleaving kininogens - multifunctional proteins derived mainly from $\alpha_{2}$-globulins. Tissue kallikrein 1 (KLK1) is synthesized in many organs, including kidney and arteries, where it can generate the vasodilators bradykinin and kallidin, which are rapidly hydrolyzed to inactive products by a group of peptidases known as kininases (Figure 1).

Kallikreins are encoded by a variable number of genes in different mammalian species. The human tissue kallikreins are encoded by a cluster of 15 genes located on chromosome 19q13.4, a position analogous to that of the kallikrein gene family 